\title{
Evolução do estado nutricional de crianças submetidas a um programa de suplementação alimentar em município do Estado de São Paulo'
}

\author{
Evolution of the nourishing condition on children \\ submitted to additional feeding program in \\ county of state of São Paulo, Brazil
}

Luciana Gonçalves CARVALHO²

Silvia Regina Dias Medici SALDIVA ${ }^{3}$

Tereza Etsuko da Costa ROSA ${ }^{3}$

Doris Lucia Martini LEI ${ }^{3}$

\section{R E S U M O}

\section{Objetivo}

O objetivo deste estudo foi avaliar o impacto do Programa de Incentivo ao Combate às Carências Nutricionais no município de Assis, estado de São Paulo.

\section{Métodos}

Foram acompanhadas 132 crianças menores de 24 meses, que permaneceram por um ano no Programa de Incentivo ao Combate às Carências Nutricionais. O estado nutricional foi avaliado pelo índice Peso para Idade expresso em escore-Z da mediana da população de referência do Nacional Center Health Statistics no início, aos 2, 6, 9 e 12 meses de acompanhamento.

\section{Resultados}

Entre as crianças estudadas, 70\% foram classificadas como desnutridas ou em risco nutricional no momento do ingresso no Programa Incentivo ao Combate às Carências Nutricionais. Em relação ao incremento de peso, comparando-se o início e após 12 meses, 64\% das crianças ganharam peso. Analisando-se a variação média do escore-Z de Peso para Idade nos intervalos de tempo estudados, segundo as faixas etárias e o estado nutricional na entrada ao programa, observou-se que as crianças menores de um ano de idade foram as que apresentaram um significativo incremento de peso.

\footnotetext{
1 Artigo baseado na dissertação de L.G. CARVALHO, intitulada "Evolução do estado nutricional de crianças submetidas a um programa de suplementação alimentar do município de Assis. Programa de Pós-Graduação em Ciências, Secretaria do Estado da Saúde de São Paulo; 2005.

2 Secretaria Municipal de Saúde de Assis. Assis, SP, Brasil.

3 Instituto de Saúde, Secretaria do Estado da Saúde do Estado de São Paulo. R. Santo Antônio, 590, $3^{\circ}$ andar, Bela Vista, 01314-000, São Paulo, SP, Brasil. Correspondência para/Correspondence to: S.R.D.M. SALDIVA. E-mail: <smsaldiva@isaude.sp.gov.br>.
} 
208 | L.G. CARVALHO et al.

\section{Conclusão}

Pode-se concluir que as crianças com déficits nutricionais e menores de um ano de idade foram as que mais se beneficiaram do programa de suplementação alimentar Incentivo ao Combate às Carências Nutricionais.

Termos de indexação: Antropometria. Avaliação Nutricional. Suplementação alimentar. Transtornos da nutrição infantil.

\section{A B S T R A C T}

\section{Objective}

The objective of this study was to assess the impact of the Program Incentive to Fight Nutritional Deficiencies in the city of Assis, state of São Paulo.

\section{Methods}

A total of 132 children under 24 months who participated in the Program Incentive to Fight Nutritional Deficiencies for one year were followed. Their nutritional status was determined by comparing their weightfor-age in z-scores at baseline and after 2, 6, 9 and 12 months of follow-up to that of the reference population median given by the National Center for Health Statistics.

\section{Results}

Most (70\%) of the children were classified as undernourished or at risk of malnutrition when they joined the Program Incentive to Fight Nutritional Deficiencies. After 12 months in the program, 64\% of the children presented an increase in their weight-for-age z-score. Analysis of the mean weight-for-age z-score variation over time, according to age group and nutritional status at baseline, showed that children under 12 months of age had a significant increase in weight-for-age z-score.

\section{Conclusion}

Malnourished children under one year of age were the ones who benefited most from the Program Incentive to Fight Nutritional Deficiencies.

Indexing terms: Anthropometry. Nutrition assessment. Supplementary feeding. Child nutrition disorders.

\section{N T R O D U ÇÃ O}

A leitura comparativa dos estudos efetuados nos últimos 22 anos em âmbito nacional e microrregional ${ }^{1-3}$, possibilita inferir um declínio marcante na prevalência da taxa global de desnutrição em crianças menores de cinco anos, passando de 18,4\% para 7,1\%. Já na Região Sudeste, nos mesmos estudos, a prevalência de desnutrição declinou de 13,4\% para 4,1\%. Recentemente, essa tendência ao declínio se confirma com os dados da Pesquisa de Orçamentos Familiares (POF 2002-2003), que mostraram uma prevalência nacional de déficit ponderal de $4,6 \%$, e de $3,6 \%$ para a Região Sudeste ${ }^{4}$.

De acordo com Benício \& Monteiro ${ }^{5}$, o declínio da desnutrição no Brasil, no período de 1989-1996, possivelmente sofreu mais influência das mudanças positivas nos serviços básicos de saúde, na escolaridade materna e nos progressos ocorridos na área de saneamento básico (fornecimento de água, infra-estrutura urbana) do que das mudanças na renda familiar, que foram mínimas neste período.

Apesar da melhora da condição nutricional das crianças, a desnutrição infantil continua ainda a ser um problema de saúde publica, devido à sua magnitude e às suas conseqüências negativas $^{6,7}$.

As ações que combatam eficientemente a pobreza são de enorme valia para a luta contra a desnutrição ${ }^{8}$. As tentativas de controlar a desnutrição como um problema de saúde pública, nos países em desenvolvimento, começaram no período pós-guerra e consistiam, especialmente, no aumento da produção dos alimentos e de programas de alimentação e educação nutricional $^{9}$. Desde então, a questão da Alimentação e Nutrição vem sendo tratada no âmbito das 
Políticas Sociais Compensatórias, em grande parte com medidas de caráter assistencialista, restringindo-se à distribuição de gêneros alimentícios para reduzir a desnutrição infantil.

Neste âmbito, dentro dos Programas de Suplementação Alimentar, foi criado pelo Ministério da Saúde, em 1998, o Programa de Incentivo ao Combate às Carências Nutricionais $(\mathrm{ICCN})^{10}$.

Este estudo visa a avaliar a efetividade do ICCN no município de Assis, investigar a evolução nutricional das crianças atendidas no programa durante 12 meses, bem como analisar algumas variáveis que interferiram nesta evolução. Este trabalho justifica-se pela necessidade de avaliar os resultados de um programa de suplementação alimentar como estratégia de recuperação nutricional em crianças menores de 24 meses, e contribuir com a discussão da implantação e do acompanhamento de programas de suplementação alimentar.

\section{MÉ T O D O S}

O Município de Assis (SP) foi qualificado no ICCN segundo a Portaria do Ministério da Saúde n 3257, de 28 de junho de 1998, sendo implantado em todas as Unidades Básicas de Saúde (UBS) e nas Unidades Saúde da Família (USF). O Município de Assis foi escolhido como local de estudo por se tratar de um município onde a principal autora ${ }^{11}$ deste artigo participou da implantação do Programa, do treinamento e da supervisão dos profissionais responsáveis pelas tomadas e registros dos dados antropométricos, e ainda do acompanhamento do referido programa no período. Seguindo as normas do Programa $\mathrm{ICCN}^{10}$, cada criança recebeu mensalmente 10 pacotes de $400 \mathrm{~g}$ ( $4 \mathrm{~kg}$ por criança/mês) de leite em pó integral e 1 lata de óleo de soja com o objetivo de contribuir para a diminuição das deficiências energético-protéicas. Os alimentos foram distribuídos nas UBS e USF onde as crianças estavam inscritas. As mães receberam orientação da equipe de saúde sobre a forma adequada de preparo do leite e do óleo de soja e as famílias receberam visitas domiciliares de acompanhamento.

Para atender aos objetivos propostos foi realizado um estudo longitudinal, por um período de 12 meses de acompanhamento, de crianças de 0 a 24 meses de idade de todas as UBS e das USF do referido município, matriculadas no ICCN.

Foram inscritas neste Programa, no período de março de 1999 a novembro de 2001, 156 crianças menores de 24 meses. Destas, 15\% foram excluídas do estudo: 20 por abandono, duas por mudança de município, uma criança com síndrome neurológica e uma criança filha de mãe HIV positivo. Portanto, fizeram parte da pesquisa 132 crianças que permaneceram por, pelo menos, 12 meses de acompanhamento no ICCN, no Município de Assis (SP).

As equipes, compostas por Auxiliares de Enfermagem e Agentes Comunitários que atuavam na área de pediatria nas unidades básicas de saúde, receberam capacitação para as tomadas de peso e altura (comprimento/estatura) conforme as técnicas preconizadas pela Organização Mundial da Saúde ${ }^{12}$.

Para a medição do peso utilizaram-se balanças pediátricas marca Filizola com capacidade de até $16 \mathrm{~kg}$ e escala dividida em 10 gramas, e para as crianças maiores de 24 meses, balanças do tipo plataforma, marca Filizola com capacidade de $150 \mathrm{~kg}$ e escala de divisão de 50 gramas. Para garantir a padronização das medidas de peso foram realizados treinamentos supervisionados para evitar falhas sistemáticas de pesagem.

Quanto à mensuração de estatura, embora ela tenha sido realizada, o instrumento utilizado (régua antropométrica da balança) não é considerado adequado para avaliar o crescimento infantil e, portanto, a altura não foi analisada nesta pesquisa.

A equipe treinada entrevistou as mães para a coleta de informações relativas à renda, à 
210 | L.G. CARVALHO et al.

escolaridade e ao número de pessoas residentes no domicílio. O peso e o comprimento ao nascer foram obtidos por meio da Declaração de Nascido-Vivo (DNV), preenchida na maternidade e, em dois casos, pela informação da mãe.

A fim de caracterizar o crescimento ponderal das crianças, foi utilizado o índice antropométrico Peso/ldade (P/I), tomando como base o padrão de referência do National Center for Health Statistics (NCHS) ${ }^{13}$, adotado pela Organização Mundial de Saúde ${ }^{14}$ e pelo Ministério da Saúde.

Neste estudo, os resultados foram expressos em escore-Z e, para avaliar o estado nutricional e a evolução nutricional foi utilizada a seguinte classificação: Desnutrido $=<-2 Z ;-2 Z<$ Risco Nutricional <-1Z; Eutrófico $>=-1 Z^{15}$.

Adotaram-se como efeito positivo de incremento de peso, nos intervalos de tempo, os valores superiores a zero escore-Z $(0,0)$.

O banco de dados foi gerado em Epi Info versão 6.0 e a avaliação nutricional por meio do aplicativo Epinut, um dos módulos do Epi Info, versão $6.9^{16}$

Para corrigir o Efeito de Regressão à Média (ERM) foi feito um ajuste de escore-Z das medidas iniciais de peso de cada criança ${ }^{17,18}$, controlando-se, assim, a influência de possíveis erros sistemáticos na obtenção do peso.

Com o objetivo de realizar uma apreciação mais detalhada das diferenças de ganho de peso ao longo dos 12 meses de intervenção, realizou-se uma comparação entre os valores médios do escore-Z da variável Peso/ldade observados nos tempos: inicial, aos 2, 6, 9 e 12 meses de programa. Para localizar as diferenças entre as médias do escore-Z de P/I no tempo, adotou-se um modelo de variância com medidas repetidas ${ }^{19}$, considerando-se os fatores: meses de intervenção, três categorias do estado nutricional e duas faixas etárias.

Para as tabelas de freqüência e para as análises de associações estatísticas, recorreu-se ao Programa SPSS versão 8 para Windows ${ }^{20}$.

O projeto foi aprovado no Comitê de Ética em Pesquisa do Instituto de Saúde, número 003/2003, em 21 de agosto de 2004.

\section{RESULTADOS}

Foram acompanhadas 132 crianças sendo a maioria do sexo feminino (71\%) e mais da metade $(57,6 \%)$ com idades entre 6 a 12 meses. Nesta população 45\% das crianças possuíam peso ao nascer adequado, 33\%, peso insuficiente (2500g-2999g) e 22\%, baixo peso (<2500g). Em relação ao estado nutricional à admissão ao programa, observou-se que entre os meninos a prevalência de desnutrição foi de $50 \%$ e entre as meninas de 24,4\%; por outro lado, a prevalência do risco nutricional entre as meninas foi de $57,4 \%$, superior a dos meninos (36,8\%) (Tabela 1).

Tabela 1. Distribuição percentual de crianças beneficiárias do Programa de Incentivo ao Combate às Carências Nutricionais, segundo o estado nutricional (Peso/ldade) à admissão, o sexo e a faixa etária. Assis (SP), 1999-2001.

\begin{tabular}{|c|c|c|c|c|c|c|c|c|}
\hline \multirow{3}{*}{ Idade (meses) } & \multicolumn{8}{|c|}{ Estado nutricional (peso/idade segundo escore-Z) } \\
\hline & \multicolumn{2}{|c|}{ Desnutrido $(<=-2 Z)$} & \multicolumn{2}{|c|}{ Risco (>-2Z e <-1Z) } & \multicolumn{2}{|c|}{ Eutrófico $(>=-1 Z)$} & \multicolumn{2}{|c|}{ Total } \\
\hline & $\mathrm{n}$ & $\%$ & $n$ & $\%$ & $\mathrm{n}$ & $\%$ & $\mathrm{n}$ & $\%$ \\
\hline \multicolumn{9}{|l|}{ Masculino } \\
\hline $6-11,9$ & 9 & 45,0 & 6 & 30,0 & 5 & 25,0 & 20 & 100,0 \\
\hline $12-24,0$ & 10 & 55,6 & 8 & 44,4 & - & - & 18 & 100,0 \\
\hline Total & 19 & 50,0 & 14 & 36,8 & 5 & 13,2 & 38 & 100,0 \\
\hline \multicolumn{9}{|l|}{ Feminino } \\
\hline $6-11,9$ & 13 & 23,2 & 33 & 58,9 & 10 & 17,9 & 56 & 100,0 \\
\hline $12-24,0$ & 10 & 23,3 & 21 & 55,3 & 7 & 18,4 & 38 & 100,0 \\
\hline Total & 23 & 24,4 & 54 & 57,4 & 17 & 18,1 & 94 & 100,0 \\
\hline
\end{tabular}


Ao analisar o perfil materno, observou-se que $59,0 \%$ das mães concluíram o ensino fundamental e apenas uma era analfabeta. Metade das famílias das crianças estudadas era composta por, no máximo, quatro pessoas. A renda per capita, classificada segundo o salário-mínimo da época mostrou que 73\% das famílias viviam com menos da metade do salário-mínimo per capita mensal, ressaltando-se, ainda, que um número considerável $(23,5 \%)$ não dispunha de um quarto do salário-mínimo (Tabela 2).

Os resultados da Tabela 3 mostram que $64 \%$ das crianças apresentaram uma evolução positiva do ganho de peso obtido pelo índice Peso/ldade, após 12 meses de intervenção, indicando algum grau de catch up em relação ao padrão de referência adotado. O percentual de crianças com incremento positivo de peso aumentou consideravelmente entre aquelas que foram classificadas como desnutridas e entre aquelas com risco nutricional na admissão ao programa (cerca de $70,0 \%)$, em comparação com as eutróficas $(36,4 \%)$, indicando associação significante entre estado nutricional no início do programa e incremento de peso ao final de 12 meses de intervenção $(p=0,014)$.

Ainda na Tabela 3, observa-se que a idade da criança no momento da matricula não interferiu no incremento de peso no decorrer de um ano de suplementação $(p=0,618)$.

Entretanto, na análise estratificada por faixa etária, observa-se que a influência do estado nutricional sobre o incremento de peso após 12

Tabela 2. Distribuição percentual de crianças beneficiárias do Programa de Incentivo ao Combate às Carências Nutricionais, segundo os indicadores socioeconômicos e demográficos de suas famílias. Assis (SP), 2001.

\begin{tabular}{lcc}
\hline Indicadores & $n$ & $\%$ \\
\hline Escolaridade materna & 62 & 47,0 \\
$1^{\circ}$ Grau Incompleto & 16 & 12,1 \\
$1^{\circ}$ Grau Completo & 23 & 17,4 \\
$2^{\circ}$ Grau Incompleto & 28 & 21,2 \\
$2^{\circ}$ Grau Completo & 1 & 0,8 \\
$3^{\circ}$ Grau Incompleto & 1 & 0,8 \\
Analfabeta & 132 & 100,0 \\
\hline Total & & \\
\hline Número de moradores no domicílio & 32 & 24,2 \\
$<=3$ & 37 & 28,0 \\
4 & 49 & 37,1 \\
5-6 & 14 & 10,6 \\
\hline$=7$ & 132 & 100,0 \\
\hline Total & & \\
\hline Renda familiar per capita (SM) & 31 & 23,5 \\
0,00 - 0,24 & 132 & 100,0 \\
0,25 - 0,49 & 65 & 49,5 \\
0,50 - 0,74 0,75 & 23 & 17,5 \\
\hline Total & 13 & 9,8 \\
\hline & & \\
\hline
\end{tabular}

SM: salário-mínimo

Tabela 3. Distribuição percentual de crianças beneficiárias ao Programa de Incentivo ao combate às carências nutricionais, de acordo com o incremento de peso após 12 meses de intervenção, segundo o indicador Peso/ldade (P/l) em escore-Z e faixa etária. Assis (SP), 1999-2001.

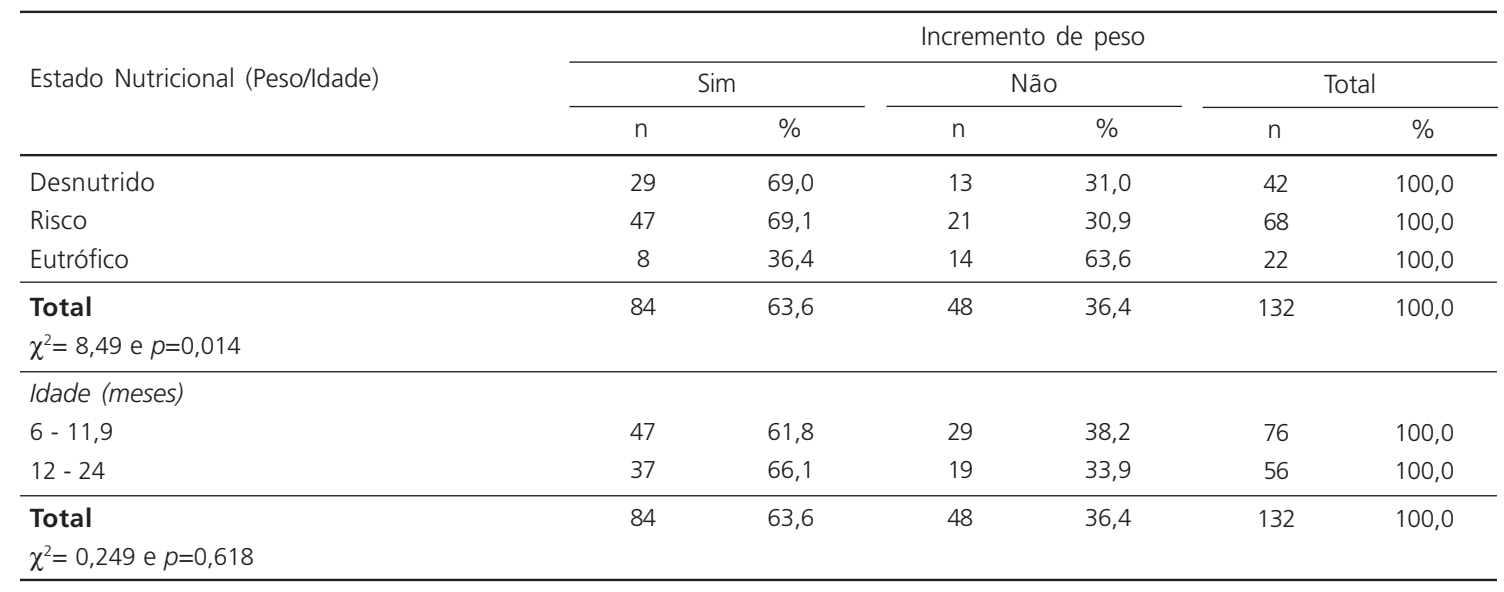


212 | L.G. CARVALHO et al.

Tabela 4. Distribuição percentual de crianças beneficiárias ao Programa de Incentivo ao Combate às Carências Nutricionais, segundo o incremento de peso após 12 meses de intervenção, o estado nutricional e a faixa etária na matrícula. Assis (SP), 1999-2001.

\begin{tabular}{|c|c|c|c|c|c|c|c|}
\hline \multirow{3}{*}{$\begin{array}{l}\text { Categorias de } \\
\text { Idade (meses) }\end{array}$} & \multirow{3}{*}{$\begin{array}{l}\text { Estado Nutricional } \\
\text { na matrícula }\end{array}$} & \multicolumn{6}{|c|}{ Incremento de peso } \\
\hline & & \multicolumn{2}{|c|}{ Sim } & \multicolumn{2}{|c|}{ Não } & \multicolumn{2}{|c|}{ Total } \\
\hline & & $n$ & $\%$ & $\mathrm{n}$ & $\%$ & $\mathrm{n}$ & $\%$ \\
\hline \multirow[t]{3}{*}{$6-11,9 m$} & Desnutrido & 16 & 72,7 & 6 & 27,3 & 22 & 100,0 \\
\hline & Risco & 28 & 71,8 & 11 & 28,2 & 39 & 100,0 \\
\hline & Eutrófico & 3 & 20,0 & 12 & 80,0 & 15 & 100,0 \\
\hline Total & & 47 & 61,8 & 29 & 38,2 & 76 & 100,0 \\
\hline \multicolumn{8}{|l|}{$\chi^{2}=13,871$ e $p=0,001$} \\
\hline \multirow[t]{3}{*}{$12-17,9 m$} & Desnutrido & 13 & 65,0 & 7 & 35,0 & 20 & 100,0 \\
\hline & Risco & 19 & 65,5 & 10 & 34,5 & 29 & 100,0 \\
\hline & Eutrófico & 5 & 71,4 & 2 & 28,6 & 7 & 100,0 \\
\hline Total & & 37 & 66,1 & 19 & 33,9 & 41 & 100,0 \\
\hline$\chi^{2}=0,104$ e $p=0,949$ & & & & & & & \\
\hline
\end{tabular}

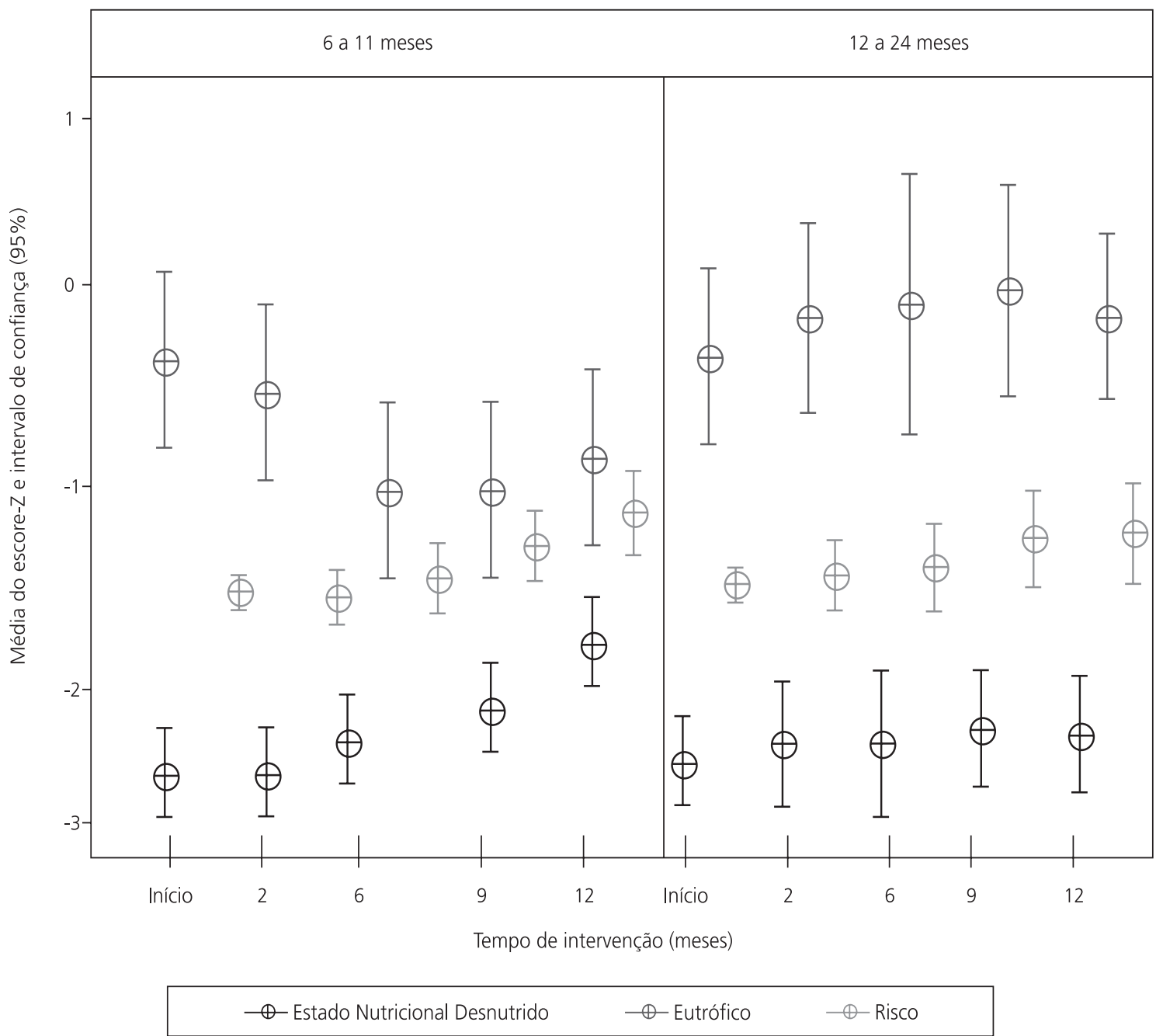

Figura 1. Distribuição das médias de Peso/ldade em escore-Z de crianças beneficiárias do Programa de Incentivo ao Combate ás Carências Nutricionais, de acordo com faixa etária e o estado nutricional distribuídos nos intervalos de tempo do estudo. Assis (SP), 1999-2001. 
meses de intervenção foi significativa somente entre as crianças menores de um ano de idade $(p=0,001)$. Ou seja, nessa faixa etária, as crianças desnutridas e em risco nutricional obtiveram percentuais significantemente maiores de incremento de peso do que as eutróficas. Em compensação, entre as crianças mais velhas este efeito não foi observado, pois o estado nutricional no ingresso ao programa não se mostrou associado ao incremento de peso durante o período de suplementação (Tabela 4).

A Figura 1 sintetiza graficamente o comportamento das médias do Peso/Idade em escore-Z de acordo com as faixas etárias e o estado nutricional nos intervalos de tempo estudados.

Ao analisar os incrementos médios de escore-Z do P/l, observou-se, que as crianças de uma maneira geral obtiveram incrementos médios positivos em todos os intervalos de tempo estudados. Não obstante, somente as crianças menores de 12 meses, e entre elas, as desnutridas e as de risco nutricional foram as que apresentaram uma diferença significante de peso após 9 meses de intervenção ( $p=0,008$ e $p=0,012$, respectivamente). Vale também assinalar, que para as crianças eutróficas, neste mesmo grupo etário, o comportamento dos incrementos médios de escore-Z do $\mathrm{P} / \mathrm{l}$ foi inusitado, uma vez que ele foi negativo ao longo do programa, embora as diferenças não tenham alcançado significância estatística.

\section{DISCUSSÃO}

Primeiramente, é preciso fazer alguns comentários sobre as limitações dos resultados deste estudo. Kirkwood et al. ${ }^{21}$ sustentam que o estudo randomizado duplo cego e controlado é o modelo padrão ouro nas avaliações de intervenções, sendo as inferências de seus resultados passíveis de maior credibilidade. Nas avaliações de programas de suplementação alimentar, sem um grupo controle concomitante, as associações encontradas entre a suplementação e as modificações no estado nutricional do grupo alvo da intervenção não podem ser inferidas como relações causais. Considerando-se que a modificação detectada esteja ocorrendo devido a outros fatores não abordados no estudo, as associações eventualmente encontradas podem ser espúrias $^{17,22}$. Como este estudo não conta com um grupo controle, como recomendado, é importante reconhecer as limitações em relação aos resultados aqui discutidos.

No entanto, por razões principalmente éticas, grande parte do que se conhece sobre o efeito da suplementação alimentar foi definida por estudos deste tipo ${ }^{17,18,23}$, procurando-se, portanto, discutir os resultados aqui encontrados enfatizando diferenças e similaridades com essa literatura.

Não fez parte do escopo deste estudo analisar os fatores associados à desnutrição, entretanto é relevante mencionar alguns aspectos que podem confirmar os efeitos prejudiciais das condições socioeconômicas sobre a saúde e nutrição destas crianças e, portanto a importância da atenção à saúde para esse grupo ${ }^{24}$, assim como atestar o valor dos princípios que regem um programa como o ICCN.

Para que uma criança atinja os padrões internacionais de crescimento é necessário que a família receba cerca de 1,5 salário-mínimo per capita ${ }^{25}$, e ao aplicar este critério, aproximadamente $90 \%$ das famílias estudadas apresentaram renda per capita inferior a 3/4 do salário mínimo o que, como foi afirmado anteriormente, não chega à metade do que é considerado satisfatório para assegurar o crescimento adequado das crianças.

A quantidade de pessoas residindo no mesmo domicílio, bem como a existência de muitos filhos em famílias de baixa renda, foram ressaltados por instituições internacionais como a United Nations for Developing People ${ }^{26}$ e a Organização das Nações Unidas para a Agricultura e a Alimentação ${ }^{27}$ como condições estreitamente associadas ao risco nutricional, especialmente nos países subdesenvolvidos. Neste estudo um pouco menos da metade das famílias (48\%) eram constituídas por cinco pessoas ou mais, residentes no mesmo domicílio, e essa situação, aliada aos baixos níveis 
214 L.G. CARVALHO et al.

de renda encontrados, pode ser tomada como condição favorável ao risco nutricional.

O reduzido nível de escolaridade das mães também pode ser apontado como um indicador do baixo nível socioeconômico destas famílias e pode retratar a reduzida capacidade das mesmas de conduzir seus filhos a um bom estado de saúde e ao crescimento e desenvolvimento adequados ${ }^{28}$.

Neste estudo, o percentual de crianças com baixo peso ao nascer foi elevado, o que de certa forma é esperado, tratando-se de uma população com características consideradas de risco ${ }^{23}$. Em relação à distribuição das crianças por sexo, o estudo revelou uma predominância da desnutrição entre os meninos, o que ratifica os achados de alguns estudos realizados com clientela de programas de recuperação nutricional ${ }^{29,30}$.

Em relação à faixa etária, a literatura enfatiza que a idade da criança no início da suplementação é um fator determinante da sua capacidade de resposta à intervenção, sendo a resposta tanto maior quanto mais precocemente se dá a intervenção ${ }^{23,31,32}$. Nesse sentido, é importante ressaltar que, no momento da matrícula, todas as crianças da amostra apresentaram entre 6 e 23 meses de idade, e que, aproximadamente, $60 \%$ das crianças eram menores de 12 meses, revelando o cumprimento da Norma Técnica que estabelecia os critérios de ingresso no ICCN.

Como recomendado, e considerando-se os aspectos de facilidade na operacionalização, na identificação das modificações rápidas e intensas em curtos intervalos de tempo ${ }^{23,33} \mathrm{O}$ indicador Peso/ldade (P/I) foi empregado para avaliar o estado nutricional das crianças.

No ingresso ao programa foi encontrada maior prevalência de crianças em risco nutricional seguido de crianças desnutridas e eutróficas. Resultados semelhantes foram observados num estudo avaliativo do mesmo programa em Mogi das Cruzes, também no interior de São Paulo ${ }^{34}$, provavelmente pela semelhança da população infantil dos dois municípios.

No decorrer de 12 meses de intervenção, em termos globais, observou-se que $64 \%$ das crianças apresentaram uma evolução favorável no índice de Peso/ldade.

Embora o Município de Assis apresente características bastante distintas das de Diadema, município de grande porte da Região Metropolitana de São Paulo, um estudo análogo ali realizado na década de $80^{35}$, sobre os efeitos da suplementação alimentar no decorrer de 12 meses de intervenção, mostrou resultados semelhantes de incremento de peso médio aos observados no presente estudo.

Já no município do Rio de Janeiro, resultados mais expressivos foram encontrados por Castro $^{18}$, que observou melhora em $75,3 \%$ da população atendida ao final de um ano e incremento médio de $+0,46$ escore- $Z$. A população-alvo era composta de crianças em faixa etária idêntica à da população aqui estudada, porém aquelas haviam ingressado no programa com Peso/ldade abaixo do percentil 3, o que pode explicar a diferença no ganho de peso.

Vários autores destacam que as crianças que ingressam em programas com piores condições nutricionais são as que mais se beneficiam da suplementação alimentar18,29,32,36. O presente estudo confirma tais evidências constatando-se que, entre as crianças desnutridas ou em risco nutricional na entrada ao programa, o percentual de crianças com incremento de peso foi significativamente maior em relação às eutróficas.

A literatura aponta a idade da criança no início da suplementação como outro fator determinante na resposta à intervenção, entretanto, no que se refere ao incremento de peso após os 12 meses, os resultados ora obtidos contrariam os achados anteriores. Ou seja, aqui, não houve diferenças nas proporções de crianças favorecidas nos diferentes grupos etários.

Outro resultado que contraria parcialmente a literatura antes referida chamou a atenção. Em análise estratificada por faixa etária, a associação verificada entre comprometimento nutricional na admissão e o incremento de peso não se manteve para o grupo de crianças maiores de 12 meses. 
Esses resultados indicam que no grupo etário de 12 a 24 meses outros fatores, que não o estado nutricional na entrada ao programa, podem influenciar no incremento de peso.

Ao analisar separadamente cada categoria de condição nutricional inicial, observou-se que os incrementos médios se comportaram de maneira diferenciada em cada intervalo de tempo. Entre as crianças desnutridas e entre as de risco nutricional, o ganho de peso foi significativo a partir do nono mês de intervenção. Esses resultados concordaram parcialmente com os achados de Lei \& Szarfarc ${ }^{36}$, os quais relatam que $80 \%$ das crianças estudadas se recuperaram do déficit ponderal, em avaliação feita após seis meses de programa.

Entre as crianças classificadas como eutróficas, o comportamento do incremento médio do escore-Z do P/I foi distinto, pois elas apresentaram decréscimo durante este período, sem, contudo, alterar a condição de eutrofia. Para uma possível explicação desse fato poderiam ser considerados os seguintes aspectos: 1) essas crianças foram aceitas no programa, mesmo eutróficas, por pertencerem a famílias consideradas com maior risco social (pais desempregados, pai ausente ou presidiário); 2) como as crianças não apresentavam risco nutricional, os responsáveis podem não ter priorizado o provimento do alimento para ela; 3 ) por elas pertencerem a famílias numerosas a diluição do alimento recebido pode ter sido maior.

Nas idades acima de 12 meses, os incrementos não foram estatisticamente significantes, considerando-se o estado nutricional inicial. Ao contrário, nessa faixa etária, entre as eutróficas, até o sexto mês de intervenção, o incremento do $\mathrm{P} / \mathrm{f}$ foi significante e diferenças essenciais não foram observadas após esse intervalo de tempo.

Conceitualmente, vários fatores não ligados à assistência desenvolvida pelo ICCN podem ter influenciado a evolução nutricional do grupo estudado, tais como mudanças favoráveis de natureza socioeconômica e na dinâmica familiar dessas crianças, fatores reconhecidamente capazes de influir sobre a saúde e a nutrição infantil ${ }^{37}$. É importante destacar que não se dispõe de evi- dências empíricas em relação a esses outros fatores, pois este estudo não avaliou as mudanças econômicas que eventualmente possam ter ocorrido entre as famílias.

Por outro lado, pode-se supor também que os grupos de orientação nutricional, a vigilância do crescimento e desenvolvimento das crianças realizada por meio do cartão da criança, o incentivo ao aleitamento materno, o acompanhamento médico e o controle de imunização, algumas das ações desenvolvidas nas UBS e nas USF, tenham tido efeito favorável, juntamente com a suplementação, na evolução do estado nutricional das crianças atendidas.

O presente estudo confirmou a relativa importância da implantação de programas de suplementação alimentar, especialmente para crianças com desnutrição grave. Embora não tenha feito parte do escopo deste trabalho analisar as ações desenvolvidas junto ao ICCN, o envolvimento direto de uma das pesquisadoras como membro da equipe de saúde responsável pelo programa permitiu levantar a seguinte hipótese: a efetividade dos programas de suplementação depende não só do provimento e da distribuição do alimento propriamente, mas também das ações desenvolvidas junto ao programa de suplementação (atenção especial às crianças desnutridas e às suas famílias pela equipe de saúde, além de visitas domiciliares freqüentes que acompanham a apreensão pelos familiares no que diz respeito às orientações realizadas). Este aspecto, como mais um determinante do sucesso no tratamento ambulatorial do desnutrido, foi confirmado em outro achado ${ }^{38}$, segundo o qual a recuperação nutricional dependeu também do compromisso assumido pela equipe de saúde junto à família e do envolvimento das mães com a equipe de saúde para que houvesse um maior investimento nas ações visando ao bem estar de seus filhos.

Desta forma, este trabalho pode contribuir para nortear não só ações dos gestores de Saúde na implantação de programas de suplementação alimentar como também das equipes de saúde envolvidas com a recuperação nutricional. 
216 | L.G. CARVALHO et al.

\section{A GRADECIMENTO}

À professora Carmem Diva Saldiva de André, do Instituto de Matemática e Estatística (IME) da Universidade de São Paulo.

\section{COLABORADORES}

L.G. CARVALHO coordenou o projeto de pesquisa, que foi tema de sua dissertação de mestrado. S.R.D.M. SALDIVA, co-orientadora, elaborou o roteiro do artigo, realizou a análise de dados e redigiu o texto. T.E.C. ROSA colaborou com o roteiro do artigo, com a análise de dados e participou da redação do texto final. D.L. LEl, orientadora, idealizou o projeto de pesquisa e colaborou com a redação do manuscrito.

\section{REFERÊ NCIAS}

1. Fundação Instituto Brasileiro de Geografia e Estatística. Fundo das Nações Unidas para a Infância. Perfil estatístico de crianças e mães do Brasil. Características sócio-demográficas, 1970-1977. Rio de Janeiro: IBGE; 1982.

2. Pesquisa Nacional de Saúde e Nutrição. Perfil de crescimento da população brasileira de 0 a 25 anos. Brasília: Instituto Nacional de Alimentação e Nutrição; 1990

3. Instituto Brasileiro de Geografia e Estatística. Pesquisa Nacional de Demografia e Saúde. Sociedade Civil de Bem-Estar Familiar no Brasil. Rio de Janeiro: IBGE; 1997.

4. Instituto Brasileiro de Geografia e Estatística. Pesquisa de orçamentos familiares 2002-2003. Antropometria e estado nutricional de crianças e adolescentes no Brasil. Rio de Janeiro: IBGE; 2006.

5. Monteiro CA, Benício MHD'A, Ortiz LP. Tendência secular do peso ao nascer. Rev Saúde Pública. 2000; 34 (Supl 6):26-40.

6. Benício MHDA, Monteiro CA. Desnutrição infantil nos municípios brasileiros. Risco de ocorrência. Brasília: Unicef; 1997.

7. Fundo das Nações Unidas para Infância e Adolescência. Estratégia para melhorar a nutrição de crianças e mulheres nos países em desenvolvimento. New York: Unicef; 1990.

8. Monteiro CA. Desnutrição: um desafio secular à nutrição infantil. J Ped [periódico online]. 2000 [acesso 2002 ago 25]. Disponível em: <http:// www.aleitamento.org.br/iblce/cristina.html>.
9. Monteiro CA. A dimensão da pobreza, da desnutrição e da fome no Brasil. Rev Est Avançados. 2003; 17(48):7-20.

10. Brasil. Ministério da Saúde. Incentivo ao combate às carências nutricionais: manual de orientação. Brasília; 2000.

11. Carvalho LG. Evolução do estado nutricional de crianças submetidas a um programa de suplementação alimentar do município de Assis/SP [dissertação]. São Paulo: Programa de Pós-Graduação em Ciências, Secretaria de Estado da Saúde; 2005.

12. Organización Mundial de la Salud. Medición del cambio del estado nutricional. Geneva: OMS; 1983.

13. National Center for Health Statistics. Growth curves for children, birth-18 years. Washington (DC): NCHS; 1977.

14. World Health Organization. Global database on child growth and malnutrition. Geneva: WHO; 1997.

15. World Health Organization. Physical status: the use and interpretation of anthropometry. Geneva: WHO; 1995. WHO Technical Report Series, 854.

16. Dean AG, Dean JA, Coulombier D, et al. Epi Info Version 6: A word processing database and statistics program for epidemiology on microcomputers. Centers for Disease Control and Prevention. Atlanta; 1994.

17. Taddei JAAC. Avaliação antropométrica do impacto nutricional de um programa de suplementação alimentar [tese]. São Paulo: Universidade de São Paulo; 1987.

18. Castro IRR. Efetividade da suplementação alimentar na recuperação do estado nutricional de crianças: avaliação do programa "Leite é Saúde" (PLS) no município do Rio de Janeiro [tese]. Rio de Janeiro: Faculdade de Saúde Pública; 1999.

19. Netter J, Kutner MH, Nachtsheim CJ, Wasserman W. Applied Linear Statistical Models. 4th ed. Chicago: Irwin; 1996.

20. Statistical Package for the Social Sciences. SPSS for Windows version 8.0. User's guide. Chicago; 1997.

21. Kirkwood BR, Cousens SN, Victora CG, Zoysa I. Issues in the design and interpretation of studies to evaluate the impact of community-based interventions. Tropical Med Int Health. 1997; 2(11): 1022-9.

22. Bauman KE. Research methods for community health and welfare. New York: Oxford University Press; 1980.

23. Goulart RMM. Evolução do estado nutricional de crianças submetidas a programa de suplementação alimentar e ações educativas à família no município de Itaquaquecetuba [dissertação]. São Paulo: Universidade de São Paulo; 1998. 
24. Organización Mundial de la Salud. Urbanización y sus repercusiones en la salud infantil: posibilidades de acción. Geneva: OMS; 1989.

25. Peliano AMM. Os programas de alimentação e nutrição no Brasil. In: Perfil estatístico de crianças e mães no Brasil: aspectos de saúde e nutrição: 1989. Rio de Janeiro: IBGE; 1992. p.111-21.

26. United Nations for Developing People. Human development report: 1990. New York: The United Nations; 1990.

27. Organização das Nações Unidas para a Agricultura e a Alimentação. Manejo de projetos comunitários de alimentação e nutrição: guia didático. Recife: FAO; 2000

28. Engle P, Menon P, Haddad L. Care and nutrition: concepts and measurement. Washington (DC): International Food Policy Research Institute; 1997.

29. Puccini RF, Goihman S, Nóbrega FJ. Avaliação do programa de recuperação de desnutridos do município de Embu, na região metropolitana de São Paulo. J Ped. 1996; 72(2):71-9.

30. Soares NT, Parente WG. Desnutrição e resultados de reabilitação em Fortaleza. Rev Nutr. 2001; 14(2):103-10.

31. Lei DLM. Estudo antropométrico da evolução do estado nutricional de crianças desnutridas beneficiárias de um programa de Suplementação Alimentar [dissertação]. São Paulo: Universidade de São Paulo; 1986.

32. Santos H. Recuperação nutricional como atividade do programa de assistência integral à saúde da criança: avaliação dos aspectos operacionais e de impacto nutricional em uma unidade de saúde da Secretaria de Estado da Saúde de São Paulo [dissertação]. São Paulo: Universidade de São Paulo; 1990.

33. Monteiro CA. Recentes mudanças propostas na avaliação antropométrica do estado nutricional infantil: uma avaliação crítica. Rev Saúde Pública. 1984; 18(1):56-63.

34. Goulart RMM. Evolução do estado nutricional de crianças desnutridas e em risco nutricional em programa de suplementação alimentar [tese]. São Paulo: Universidade de São Paulo; 2003.

35. Lei DLM, Monteiro CA, Lerner BR, Chaves SP. Medindo o impacto de programas de recuperação nutricional de pré-escolares: teste de uma metodologia. Rev Saúde Pública. 1989; 23(3):230-5.

36. Lei DLM, Szarfarc SC. Intervenção nutricional a desnutridos: evolução antropométrica após um ano de programa. Rev Bras Cresc Des Hum. 1992; 2:100-16.

37. Tonete VLP, Carvalhaes MABL, Trezza EMC. Evolução nutricional de crianças carentes atendidas por programa de suplementação alimentar. Pediatria (São Paulo). 2003; 25(3):101-9.

38. Yamamoto RM, Alderete JMS, Contim D, Cardoso I, Nascimento AA, Silva MLS, et al. Abordagem multiprofissional da desnutrição energético protéica em uma unidade básica de saúde: relato de uma experiência. Pediatria. 1998; 20(4):399-405.

Recebido em: 26/6/2007

Versão final reapresentada em: 11/3/2008 Aprovado em: 16/7/2008 